\title{
Advanced Thymic Carcinoma
}

National Cancer Institute

\section{Source}

National Cancer Institute. Advanced Thymic Carcinoma. NCI Thesaurus. Code C159903.

Thymic carcinoma that has spread extensively to other anatomic sites or is no longer responding to treatment. 